\title{
Simultaneous generation and compression of broadband terahertz pulses in aperiodically poled crystals
}

\author{
Koustuban Ravi ${ }^{1,2,{ }^{*}}$ And Franz X. Kärtner ${ }^{1,2,3}$ \\ ${ }^{1}$ Center for Free-Electron Laser Science, DESY, Notkestraße 85, Hamburg 22607, Germany \\ ${ }^{2}$ Research Laboratory of Electronics, Massachusetts Institute of Technology, Cambridge, MA 02139, USA \\ ${ }^{3}$ Department of Physics, University of Hamburg, Hamburg 22761, Germany \\ koustuban@alum.mit.edu
}

\begin{abstract}
We introduce a technique to generate compressed broadband terahertz pulses based on cascaded difference-frequency generation. The approach employs a non-uniform sequence of pump pulses in aperiodically poled crystals. The pump-pulse format and poling of crystals conceived are such that the emergent terahertz pulse is already compressed. The method circumvents pump-pulse distortions that result from non-collinear approaches and the need for external compression. While capable of generating even single-cycle pulses, it is particularly efficient for the generation of pulses with few to tens-of-cycles duration. For instance, calculations accounting for cascading effects predict conversion efficiencies in the few percent range for cryogenically-cooled lithium niobate. The focused electric fields are $\gg 100 \mathrm{MV} / \mathrm{m}$ in free space.
\end{abstract}

(C) 2019 Optical Society of America under the terms of the OSA Open Access Publishing Agreement

\section{Introduction}

High-field terahertz radiation are important probes for a variety of fundamental scientific investigations [1-7]. In addition, they are considered to be invaluable to future particle accelerator technology [8-13], which could in turn transform a wide swath of applications such as X-ray generation [14].

While various methods to generate terahertz $(\mathrm{THz})$ radiation, ranging from photoconductive switches [15] to gyrotrons [16] and free-electron lasers [17] exist, they have been limited in one or more of frequency, peak power or bandwidth.

With the proliferation of advanced solid-state laser technology, methods based on nonlinear frequency down-conversion of high-power infrared or near-infrared (NIR) frequencies have gained ground. Due to the large difference in terahertz and NIR photon energies, the single-photon energyconversion efficiency of this class of approaches is expected to be low. However, repeated energy down-conversion of NIR photons or cascaded difference-frequency generation (DFG) [18] may be utilized to produce optical-to-terahertz energy conversion efficiencies exceeding the percentlevel $[19,20]$. In addition, laser-driven approaches offer precise synchronization possibilities, which are instrumental for spectroscopic experiments.

Despite remarkable progress, drawbacks persist with various nonlinear frequency downconversion schemes. In organic crystals, terahertz and optical waves are close to being perfectly phase matched, purely by virtue of fortuitous material properties. Therefore, crystals shorter than the coherence length maybe utilized $[20,21]$ to generate broadband terahertz radiation $>1 \mathrm{THz}$ efficiently. However, in general, phase matching needs to be enforced by developing a suitable mechanism.

A general scheme for broadband phase matching is via the use of tilted pulse fronts [22] in non-collinear geometries. This method [23-25], amenable for broadband or single-cycle terahertz pulse generation, has produced very high conversion efficiencies and pulse energies in the $<1 \mathrm{THz}$ regime in lithium niobate $[19,26]$. 
However, the approach is ultimately limited by a spatio-temporal break up of the optical pump pulse upon generating terahertz radiation at high conversion efficiencies [27-30]. This is because, at high conversion efficiencies, cascading effects produce large spectral and angular broadening of the pump. This issue may be alleviated using a superposition of beamlets [31-33] and variants thereof $[34,35]$. Alternatively, attempts to eliminate the drawbacks of tilted pulse fronts in lithium niobate by utilizing smaller pulse-front tilt angles are being pursued [36,37].

Quasi phase matching based on periodic inversion of the second-order nonlinearity [38-43] addresses the break-up issues posed by non-collinear phase-matching geometries but is only suited to the generation of narrowband terahertz pulses. Recently, we suggested the use of aperiodically poled structures [44] to generate broadband terahertz pulses. Such structures are advantageous in that they eliminate limitations posed by non-collinear phase-matching techniques in conditions where dramatic cascading occurs. They also open up the possibility of re-using the optical pump pulse in subsequent stages. However, they require an external device to compress the chirped terahertz pulses, which increases the complexity of practical implementation.

In this paper, we present an approach previously introduced by us in $[45,46]$, which utilizes aperiodically poled nonlinear crystals to generate broadband terahertz pulses which emerge already compressed. In this regard, work by [47] similar to that initially developed in $[45,46]$ may be of interest.

Our proposal entails using aperiodically poled crystals in conjunction with a non-uniform sequence of short pump pulses. Such a non-uniform sequence may in turn be generated by the superposition of a pair of long pulses. The long pulses will be required to possess different bandwidths, a relative chirp rate and will need to be spectrally separated by the desired terahertz center-frequency.

Each individual pulse in this non-uniform sequence of pump pulses shall possess a different bandwidth (or duration). As a result, each pump pulse in the sequence generates terahertz radiation centered at a different frequency.

Furthermore, each pump pulse in the sequence will be phase matched for terahertz generation at a different location in the aperiodically poled crystal.

The crystal may therefore be poled in such a way that the time delay at which a particular terahertz frequency is generated is offset by its propagation time. For example, consider a material where the terahertz group velocity is smaller than the optical group velocity. Here, the first pump pulse in the sequence will generate terahertz radiation centered at frequency $f_{1}$ at the beginning of the crystal. The time it takes for this terahertz wave centered at frequency $f_{1}$ to reach the end of the crystal is designed to be synchronized with the time of arrival of the last pump pulse in the sequence. The last pump pulse in turn generates terahertz radiation centered at frequency $f_{2}$, which is now in phase with the terahertz wave centered at $f_{1}$. This way, every generated terahertz frequency component remains in phase to cumulatively produce a transform-limited terahertz pulse. A more detailed step-by-step explanation is provided in Section 2 following Fig. 1.

Viewed alternately, aperiodic poling imparts a chirp onto the generated terahertz pulse. However, this chirp can be exactly compensated by the relative chirp between the two aforementioned long pulses (whose superposition produces the non-uniform sequence of short pump pulses).

The above physical description is not guaranteed to hold true in the presence of cascading effects due to the spectral broadening experienced by the pump. However, in the present work, we verify the feasibility of the approach even in the presence of cascading effects. This is a key aspect of our work. In addition, it is imperative that cascading effects be considered to accurately predict energy conversion efficiencies that exceed the single-photon conversion level (or Manley-Rowe limit).

Thus, the collinear geometry of the present approach addresses the issues of pump-pulse distortion suffered by tilted pulse fronts while circumventing the need for external compression demanded by prior proposals of aperiodic structures. The approach is also promising due to 
the development of $\mathrm{cm}^{2}$-aperture, poled lithium niobate crystals with $<0.1 \mu \mathrm{m}$ control over domain periods [48]. On the other hand, the required control of poling periods in this approach are much larger, i.e. $>1 \mu \mathrm{m}$. Furthermore, the required pump pulses can be generated using recent developments in high-energy Ytterbium amplifiers [49].

It is worth noting that self-compressing second-harmonic generation has been previously demonstrated [50-53]. However, in contrast to second-harmonic generation where a single chirped pump pulse is sufficient, a non-uniform sequence of pump pulses is necessary for terahertz generation. Secondly, it is worth reiterating that terahertz generation with high conversion efficiency requires cascaded DFG. Such strongly phase-matched cascading effects are not considered in self-compressing second-harmonic generation systems.

It is found that the technique presented here is capable of generating even single-cycle pulses (See Fig. 2). However, it is particularly efficient for generating terahertz pulses of few to tens of cycles. As a specific example, the use of cryogenically-cooled lithium niobate at $80 \mathrm{~K}$ pumped by $1 \mu \mathrm{m}$ lasers are predicted to yield optical-to-terahertz energy conversion efficiencies of a few percent with peak-field strengths of $50 \mathrm{MV} / \mathrm{m}$ for collimated beams. Similar performance with materials such as Potassium Titanyl Phosphate (KTP) may also be envisaged. The use of a long sequence of pulses spanning a $100 \mathrm{ps}$ full-width at half-maximum (FWHM) total duration permits pump fluences of $1 \mathrm{Jcm}^{-2}$ [43]. For $\mathrm{cm}^{2}$-aperture, poled crystals [48], this translates to pump-pulse energies of $\sim 1 \mathrm{~J}$. For the above mentioned percent-level energy conversion efficiencies and $50 \mathrm{MV} / \mathrm{m}$ fields, this translates to terahertz pulse energies of $>10 \mathrm{~mJ}$ and focused field strengths in free space of $\gg 100 \mathrm{MV} / \mathrm{m}$. Such high-field, few to tens-of-cycles terahertz pulses have been predicted to be useful for the acceleration of relativistic electrons [54]. In particular, such terahertz pulses could be very effective in mitigating space-charge effects. Therefore, they could produce electron bunches that are much shorter compared to those produced by terahertz pulses with hundreds of cycles [43]. Such electron bunches could in turn be disruptive for X-ray generation.

We first describe the physical principle governing the operation of the proposed method. We then proceed to analytically verify its functionality. The analysis is shown to be validated by numerical simulations. Subsequently, we present analytic expressions and perform simulations in both depleted (i.e including cascading effects) and undepleted limits to describe the behavior of the system and furnish optimal parameters.

\section{Physical principle}

The proposed method requires :

(i) An aperiodically poled electro-optic crystal with a second-order nonlinearity profile $\chi^{(2)}(z)$. This allows for various longitudinal positions $z$ to be phase matched for the difference-frequency generation of different terahertz angular frequencies $\Omega_{g}(z)$.

(ii) A sequence of pulses with non-uniform duration, to each generate a different frequency.

The general idea is to compensate for the propagation times of terahertz radiation generated at various locations $z$, by the times at which their generation is initiated. This can be achieved by exploiting the difference in terahertz and optical group velocities.

Consider the typical situation when the terahertz velocity is smaller than that of the optical pump. In this case, pulses appearing earliest in the pump sequence would be phase matched for terahertz generation at the beginning of the crystal. On the other hand, pulses appearing latest in the sequence would be phase matched for terahertz generation at the end of the crystal.

The overall envelope of the optical pump would walk-off from the terahertz pulse that is generated. However, at each location $z$, a different pulse in the sequence would be overlapped with the terahertz pulse.

Furthermore, this pulse would generate precisely that angular frequency $\Omega_{g}(z)$ that is phase matched by the local poling period of $\chi^{(2)}(z)$. 


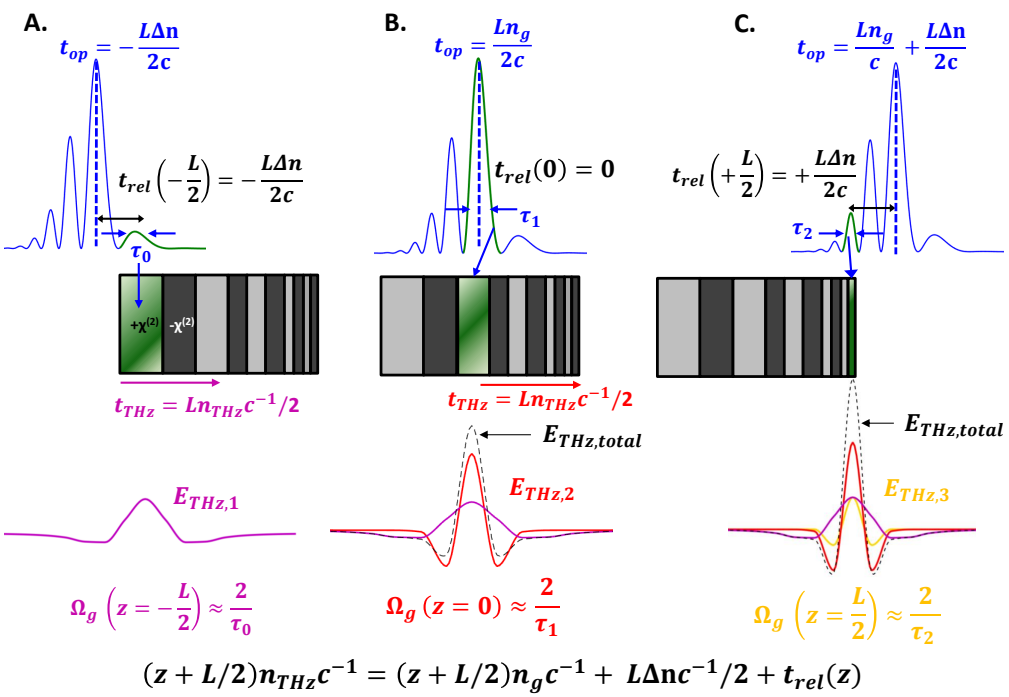

Fig. 1. Schematic of outlined approach : An aperiodically poled crystal with secondorder susceptibility $\chi^{(2)}(z)$ for the difference-frequency generation of different terahertz frequencies at different locations and a non-uniform sequence of pulses (blue) are required. The idea exploits the difference in optical and terahertz group velocities to offset the terahertz propagation time $t_{T H z}$ by the time at which it gets generated. (a) The pulse of duration $\tau_{0}$ (green outline) is phase matched at $z=-L / 2$ (green-shaded area) to generate a terahertz wavelet $E_{T H z, 1}$ of angular frequency $\Omega_{g}(z=-L / 2)=2 / \tau_{0}$ (purple trace) at a time $t_{\text {rel }}=-L \Delta n c^{-1} / 2$, relative to the center of the overall pump envelope. The terahertz wavelet born here reaches $z=0$ at $t_{T H z}=L_{T H z} c^{-1 / 2}$. (b) The central pulse in the sequence with duration $\tau_{1}$ (green outline) is phase matched at $z=0$ (green-shaded area) to generate a terahertz wavelet $E_{T H z, 1}$ with $\Omega_{g}(z=0)=2 / \tau_{1}$ (red trace). The previously generated terahertz radiation has reached $z=0$ at precisely the same time as the newly generated trace, leading to coherent growth of the total field $E_{T H z \text {,total }}$ (black-dashed). (c) The pulse with duration $\tau_{2}$ is phase matched at the end of the crystal. While the overall pump envelope slips through the terahertz field, there is always a pulse in the sequence which is overlapped with the terahertz field. This pulse generates a terahertz angular frequency $\Omega_{g}(z)$ that is precisely phase-matched at $z$. The length of the crystal here is arbitrary, hence the terahertz pulse remains compressed throughout. 


\subsection{Obtaining $t_{\text {rel }}(z)$}

The problem at hand is therefore to obtain expressions for $\Omega_{g}(z)$ and $\chi^{(2)}(z)$. This is done by first determining $t_{\text {rel }}(z)$ - the time relative to the center of the pump envelope, at which the pulse in the pump sequence, phase matched for terahertz generation at $z$ arrives.

We proceed by examining Fig. 1. Here, snapshots of the aperiodically poled crystal between $z=\mp L / 2$, the optical pump sequence (blue) and generated terahertz wavelets at different time instants $t_{o p}$ are depicted.

The time $t_{o p}$ is referenced to the center/peak of the overall pump envelope. For instance, when $t_{o p}=0$, the peak of the pump envelope is located at $z=-L / 2$.

In Fig. 1(a), the first pulse in the sequence (green-outline) is located at $z=-L / 2$. If this pulse occurs at a time $t_{\text {rel }}(-L / 2)$ relative to the center of the envelope, then based on our prior definition, $t_{o p}=t_{r e l}(-L / 2)$. The pulse (green-outline) has a duration $\tau_{0}$ that is phase matched for the generation of terahertz radiation with angular frequency $\Omega_{g}=2 / \tau_{0}$ at $z=-L / 2$ (green-shaded area). Difference-frequency generation produces a terahertz wavelet $E_{T H z, 1}$ (purple) as depicted below the crystal. The generated wavelet takes a time $t_{T H z}=L n_{T H z} c^{-1} / 2$ to reach the center of the crystal at $z=0$. Note, that the choice of the crystal center (i.e. $z=0$ ) is arbitrary and is only for illustrative purposes. We shall later find that the terahertz pulse remains compressed throughout.

In Fig. 1(b), the central pulse in the sequence of duration $\tau_{1}$ (green-outline) with $t_{r e l}=0$ is depicted. It is phase matched (green-shaded area) for the generation of the corresponding angular frequency $\Omega_{g}=2 / \tau_{1}$ at $z=0$.

For coherent growth of terahertz radiation to occur, the terahertz wavelet generated in Fig. 1(a) has to now overlap precisely with the central pulse in the envelope. This would require the time $\Delta t_{o p}$ elapsed between Figs. 1(a) and 1(b) to be the same as the time taken for the terahertz radiation to reach $z=0$ from $z=-L / 2$.

Thus, we have the condition that $\Delta t_{o p}=L n_{g} /(2 c)-t_{r e l}(-L / 2)=L n_{T H z} /(2 c)$. This yields $t_{\text {rel }}(-L / 2)=-L \Delta n /(2 c)$, where $\Delta n=n_{T H z}-n_{g}$ is the difference between terahertz and optical group refractive indices.

Upon satisfying this requirement, we see in Fig. 1(b) that the terahertz wavelet $E_{T H z, 2}$ (red) generated at $z=0$ overlaps precisely with $E_{T H z, 1}$ (purple) from Fig. 1(a) to produce coherent growth of the total field $E_{T H z \text {,total }}$ (black-dashed).

Extending the above argument to general $z$, we obtain the following constraint for $t_{r e l}(z)$ in Eq. $(1)$.

$$
\frac{(L / 2+z) n_{T H z}}{c}=\frac{(L / 2+z) n_{g}}{c}-t_{r e l}(-L / 2)+t_{r e l}(z)
$$

Substituting $t_{r e l}(-L / 2)=-L \Delta n /(2 c)$ in Eq. (1) as deduced above, we obtain the following expression for $t_{\text {rel }}(z)$ below.

$$
t_{\text {rel }}(z)=\frac{z\left(n_{T H z}-n_{g}\right)}{c}=\frac{z \Delta n}{c}
$$

The validity of Eq. (2) is seen in Fig. 1(c) which shows the optical pulse in the pump sequence with duration $\tau_{2}$ (green-outline) with $t_{r e l}=+L \Delta n /(2 c)$. This is phase matched (green-shaded area) for $\Omega_{g}=2 / \tau_{2}$ and the generated $E_{T H z, 3}$ (yellow) overlaps with the previously generated terahertz radiation at $z=L / 2$. The total terahertz field has continued to grow between Figs. 1(a)-1(c), as evident in the superposition of individual wavelets $E_{T H z \text {,total }}$ (black-dashed).

Thus, it has been described how a compressed terahertz pulse maybe generated using the proposed method. 


\subsection{Generating a non-uniform sequence of pulses}

Now, we prescribe a method to generate the desired non-uniform sequence of pulses. The overall pump field described by the electric field phasor $E(t)$ in time $t$ can be generated by the superposition of two pulses $E_{1}(t), E_{2}(t)$ as shown in Eq. (3a).

In Eq. ( $3 b), E_{1}(t)$ represents a pulse centered at angular frequency $\omega_{1}$ with transform-limited duration $\tau_{T L}$, chirped at a rate $b=\left(\tau \tau_{T L}\right)^{-1}$ to a duration $\tau \gg \tau_{T L}$. On the other hand, from Eq. (3c), $E_{2}(t)$ represents a pulse with transform-limited duration $\tau$, centered at angular frequency $\omega_{2}$. $E_{1}(t), E_{2}(t)$ and their corresponding spectra $E_{1}(\omega), E_{2}(\omega)$ in angular frequency $\omega$ are delineated in Eqs. (3b) and (3c) respectively.

$$
\begin{gathered}
E(t)=E_{1}(t)+E_{2}(t) \\
E_{1}(t)=E_{1} e^{j \omega_{1} t} e^{j b t^{2}} e^{-t^{2} / \tau^{2}} \\
E_{1}(\omega)=\frac{E_{1} e^{-j b^{-1}\left(\omega-\omega_{1}\right)^{2}} e^{-\frac{\left(\omega-\omega_{1}\right)^{2} \tau_{T L}^{2}}{4}}}{2 \sqrt{\pi\left(\tau^{-2}+j b\right)}} \\
b=\left(\tau \tau_{T L}\right)^{-1} \\
E_{2}(t)=E_{2} e^{j \omega_{2} t} e^{-t^{2} / \tau^{2}} \\
E_{2}(\omega)=\frac{\tau E_{2} e^{-\frac{\left(\omega-\omega_{2}\right)^{2} \tau^{2}}{4}}}{2 \sqrt{\pi}}
\end{gathered}
$$

From Eqs. (3b) and (3c), it is clear that $\Omega_{0}=\omega_{1}-\omega_{2}$ is defined as the central terahertz angular frequency, while $f_{0}=\Omega_{0} /(2 \pi)$ is the central terahertz frequency.

It can be seen that the overall intensity profile $|E(t)|^{2} \propto \cos ^{2}\left[\left(\Omega_{0}+b t\right) t / 2\right]$. This corresponds to a spacing between pulses given by $\approx T_{0}\left(1-b t / \Omega_{0}\right)$ (for $\left.t \ll \tau\right)$ and pulse duration $\approx T_{0}\left(1-b t / \Omega_{0}\right) / 2$, where $T_{0}=2 \pi / \Omega_{0}$ is the time period of the central terahertz frequency. Thus it is clear that for $b>0$, earlier pulses $(t<0)$ in the sequence are longer and spaced more sparsely.

\subsection{Obtaining $\Omega_{g}(z)$}

Since the generated terahertz frequency is merely the difference of the instantaneous frequencies of the two pulses, it may be deduced that $\Omega_{g}=\omega_{1}+b t-\omega_{2}=\Omega_{0}+b t$. However, at a given $z$, only terahertz frequencies corresponding to $t_{r e l}(z)$ from Eq. (2) shall be phase matched, which yields the relationship for $\Omega_{g}(z)$ in Eq. (4).

$$
\Omega_{g}(z)=\Omega_{0}+\frac{b z \Delta n}{c}
$$

\subsection{Poling profile}

Having established the spatial distribution of terahertz frequencies by Eq. (4), it is straightforward to obtain the corresponding poling profile. By analogy to a periodically poled crystal, the required aperiodic poling profile to generate $\Omega_{g}(z)$ is given by Eq. (5).

$$
\chi^{(2)}(z)=\chi_{b}^{(2)} \operatorname{sgn}\left[\cos \left(\Omega_{g}(z) \Delta n z c^{-1}\right)\right]
$$


In Eq. (5), $\chi_{b}^{(2)}$ is the second-order nonlinear bulk susceptibility and sgn represents the signum function.

\section{Analysis}

\subsection{Proof of functionality}

In this section, we analytically show that the proposed approach indeed functions as suggested. A solution for the terahertz spectral component $E_{T H z}(\Omega, z)$ at angular frequency $\Omega$ is obtained from the nonlinear wave equation by assuming a solution of the form $E_{T H z}(\Omega, z)=$ $A_{T H z}(\Omega, z) e^{-j \Omega n_{T H z} c^{-1} z}$. The evolution of the terahertz spectral envelope $A_{T H z}(\Omega, z)$ in the undepleted limit then follows [43] and is reproduced in Eq. (6) below.

$$
\frac{d A_{T H z}(\Omega, z)}{d z}=\frac{-j \Omega}{2 c n_{T H z}} \chi^{(2)}(z) e^{-j \Delta k z} \mathfrak{F}\left(|E(t, z)|^{2}\right)-\frac{\alpha(\Omega)}{2} A_{T H z}(\Omega, z)
$$

In Eq. (6), $\Delta k=-\Delta n \Omega c^{-1}$ represents the phase mismatch between the generated terahertz radiation and incident optical pump. The total pump field $E(t)$ is obtained from Eqs. (3a)-(3c). The term $\mathfrak{F}\left(|E(t)|^{2}\right)$ in Eq. (6) corresponds to the spectral distribution of the nonlinear polarization due to various difference-frequency generation processes. This term is found in previous models such as $[43,44,55]$. The final term in Eq. (6) corresponds to terahertz absorption with $\alpha(\Omega)$ being the terahertz absorption coefficient.

Upon integrating Eq. (6) over longitudinal distance $z$, we obtain the following expression for $A_{T H z}(\Omega, z)$ in Eq. (7).

$$
A_{T H z}(\Omega, z)=\frac{-j \Omega \tilde{F}\left(|E(t)|^{2}\right) e^{-\alpha z / 2} \int_{-L / 2}^{z} e^{(-j \Delta k+\alpha / 2) z^{\prime}} \chi^{(2)}\left(z^{\prime}\right) d z^{\prime}}{2 c n_{T H z}}
$$

In order to obtain a closed-form expression for $A_{T H z}(\Omega, L / 2)$ from Eq. (7) that illustrates the functionality of the approach, a few simplifying assumptions are made. These are without the loss of generality as validated later by detailed numerical calculations. First, absorption in Eq. (7) is ignored. Next, the expression for $\chi^{(2)}(z)$ from Eq. (5) is approximated as $\chi^{(2)}(z) \approx \chi_{b}^{(2)} e^{-j \Omega_{g} \Delta n c^{-1} z}$, where $\Omega_{g}(z)$ is given by Eq. (4). In addition, the limits of integration in Eq. (7) maybe set to to $[-\infty, \infty]$. This approximation maybe justified by the fact that contributions outside the interval $[-L / 2, L / 2]$ are less significant due to the finite bandwidth of the pump pulse. Upon explicit evaluation of $\mathfrak{F}\left(|E(t)|^{2}\right)$ using Eqs. (3a)-(3c), we obtain the following closed-form expression for $A_{T H z}(\Omega, L / 2)$ in Eq. (8) below.

$$
A_{T H z}(\Omega, L / 2)=\frac{\Omega \chi_{b}^{(2)} E_{1} E_{2} e^{-\frac{\left(\Omega-\Omega_{0}\right)^{2} \tau_{T L}^{2}}{2\left(4 \tau_{T L}^{2} \tau^{-2}+1\right)}}}{4 b \Delta n n_{T H z}}\left(e^{-\frac{j b\left(\Omega-\Omega_{0}\right)^{2}}{4\left(b^{2}+4 \tau^{-4}\right)}} e^{\frac{j\left(\Omega-\Omega_{0}\right)^{2}}{4 b}}\right)
$$

Inspecting the first exponential term in Eq. (8), we notice that $A_{T H z}(\Omega, L / 2)$ is centered about the terahertz angular frequency $\Omega_{0}$ with bandwidth proportional to $\left(8 \tau^{-2}+2 \tau_{T L}^{-2}\right)^{1 / 2}$. For the case when the chirped-pulse duration is much greater than the transform-limited duration, i.e. $\tau>>\tau_{T L}$, the terahertz spectral bandwidth is proportional to $\tau_{T L}^{-1}$. This corresponds to the range of possible beat frequencies between the two input pulses and is consistent with expectations.

The terms enclosed within the round brackets in Eq. (8) correspond to the various phase terms that accrue. The first phase term arises from the difference-frequency generation process, while the second term arises from the aperiodic poling profile. Notice that the two terms are of opposite signs, indicative of phase compensation. 


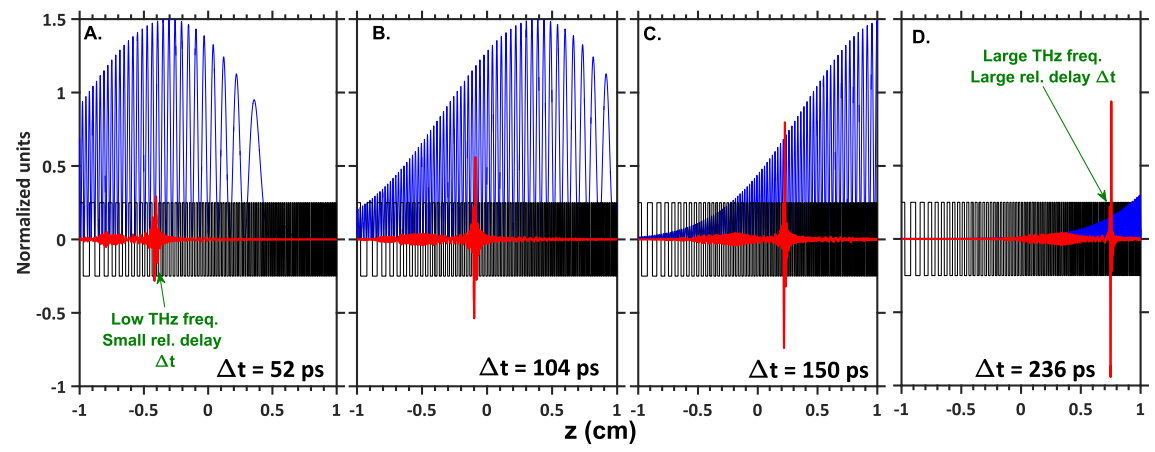

Fig. 2. Numerical simulations of Eq. (6) of a non-uniform sequence of pulses (blue) with parameters $\tau=150 \mathrm{ps}, \tau_{T L}=0.5 \mathrm{ps}$ and $f_{0}=0.5 \mathrm{THz}$ in an $L=2 \mathrm{~cm}$ lithium niobate crystal with poling profile given by Eq. (5) at $\mathrm{T}=80 \mathrm{~K}$. The poling profile (black) possesses a gradually reducing period from $910 \mu \mathrm{m}$ to $100 \mu \mathrm{m}$.(a) The lower terahertz frequencies are generated earlier, at the beginning of the crystal to minimize absorption. The overall pump envelope walks ahead of the single-cycle terahertz transient (red) due to larger group velocity. (b)-(d) The poling profile is arranged such that the terahertz frequencies are phase matched precisely at locations where the corresponding pulses in the sequence (blue) overlap with the terahertz transient (red) to produce coherent growth in the terahertz field and bandwidth.

Given that the chirp rate $b=\left(\tau \tau_{T L}\right)^{-1}$ (Eq. (3b)) and that the chirped pulse duration $\tau \gg \tau_{T L}$ , the $e^{\frac{-j b\left(\Omega-\Omega_{0}\right)^{2}}{4\left(b^{2}+4 \tau^{-4}\right)}}$ term is almost perfectly compensated by the $e^{\frac{j b\left(\Omega-\Omega_{0}\right)^{2}}{4 b^{2}}}$ term. In fact, one may achieve exact compression by adjusting the chirp rate to $b^{\prime}$ defined by Eq. (9).

$$
b^{\prime}=b \sqrt{1+4 \tau^{-4} b^{-2}}
$$

Thus, it has been analytically shown in Eq. (8) that the proposed approach produces a perfectly compressed terahertz pulse in the ideal limit.

\subsubsection{Numerical validation}

To validate our analysis, numerical simulations employing Eq. (6) without approximations are performed for a pump pulse $E(t)$ defined by Eqs. (3a)-(3c) with parameters $\tau_{T L}=0.5 \mathrm{ps}$, $\tau=150 \mathrm{ps}$ and $f_{0}=0.5 \mathrm{THz}$. The complete material dispersion in the NIR frequency range [56], terahertz frequency range [57] and terahertz absorption [58] at $T=80 \mathrm{~K}$ were taken into account. The bulk second-order nonlinear susceptibility was assumed to be $\chi_{b}^{(2)}=336 \mathrm{pm} / \mathrm{V}$. An $L=2 \mathrm{~cm}$ long lithium niobate crystal with a poling profile given by Eq. (5) is assumed. The poling period varies from $\Lambda=910 \mu \mathrm{m}$ at the beginning of the crystal to $\Lambda=100 \mu \mathrm{m}$ at the end of the crystal. The results are presented in Figs. 2(a)-2(d). The lowest terahertz frequencies are phase matched at the beginning of the crystal, evident by the larger poling periods (black), to minimize absorption.The long pulses in the pump sequence (blue) arrive first. They generate the low terahertz frequencies and walk-off from the generated terahertz transient (red) in Fig. 2(a) since $n_{T H z}>n_{g}$ in lithium niobate. However, the terahertz transient is always synchronized to a latter pulse in the pump sequence. These generate terahertz frequencies $\Omega_{g}(z)$, which also happen to be phase matched at the same location as the terahertz transient. Thus, the terahertz transient continues to grow in strength and reduce in duration in Figs. 2(b)-2(d). Thus, a compressed single-cycle terahertz transient can be generated in a manner described at the outset. 


\subsection{Length and frequency behavior}

In Eqs. (10a)-(10c), an analytic solution to Eq. (6) which accounts for the finite length of the crystal, absorption and terahertz dispersion is furnished using imaginary error functions (erfi). In comparison to a complete numerical solution of Eq. (6), these expressions only account for the principal harmonic components of the aperiodic poling profile, i.e. $\chi^{(2)}(z) \approx \chi_{b}^{(2)} e^{-j \Omega_{g} \Delta n c^{-1} z}$.

$$
\begin{gathered}
A_{T H z}(\Omega, z)=\frac{\Omega \chi_{b}^{(2)} E_{1} E_{2} e^{-\frac{\left(\Omega-\Omega_{0}\right) \tau_{T L}^{2}}{2\left(4 \tau_{T L}^{2} \tau^{-2}+1\right)}}\left(e^{-\frac{j b\left(\Omega-\Omega_{0}\right)^{2}}{4\left(b^{2}+4 \tau^{-4}\right)}} e^{\frac{j\left(\Omega-\Omega_{0}\right)^{2}}{4 b^{\prime}}}\right) G(\gamma, \theta)}{8 \sqrt{b b^{\prime}} \Delta n n_{T H z}} \\
G(\gamma, \theta)=e^{-\gamma \theta / 2}\left(\operatorname{erfi}\left[\frac{\sqrt{j}}{2}(j \gamma-\theta)\right]-\operatorname{erfi}\left[\frac{\sqrt{j}}{2}\left(\left.j \gamma\right|_{z=-\frac{L}{2}}-\theta\right)\right]\right) \\
\gamma=\frac{2 z}{z_{0}}-\frac{\Omega-\Omega_{0}}{b^{\prime 1 / 2}}, \theta=\frac{\alpha z_{0}}{2}, z_{0}=\frac{c}{b^{\prime 1 / 2} \Delta n}
\end{gathered}
$$

In Eq. (10a), terms outside the round brackets are similar to corresponding terms from Eq. (8). The phase terms within brackets correspond to those from the difference-frequency generation process and aperiodic poling profile respectively. As already shown, these tend to compensate each other. The dependence of the terahertz spectral component $A_{T H z}(\Omega, z)$ on length $z$, detuning $\Omega-\Omega_{0}$ and terahertz absorption coefficient $\alpha(\Omega)$ are captured by the function $G(\gamma, \theta)$ which is presented in Eq. (10b). In Eq. (10b), the prefactor $e^{-\gamma \theta / 2}$ corresponds to the loss term. The normalized variables $\gamma, \theta$ and $z_{0}$ are in turn defined in Eq. (10c). Note that $\gamma$ captures propagation and bandwidth effects, $\theta$ accounts for absorption and $z_{0}$ is a normalized distance parameter. Based on the properties of erfi functions, it can be readily shown that $|G| \leq 2$. In this limit, Eq. (10a) becomes identical to Eq. (8).

We plot $G(\gamma, \theta)$ from Eq. (10b) in Fig. 3 in the limit when $-L / 2 \rightarrow-\infty$. This enables us to study key features of $A_{T H z}(\Omega, z)$. Later on it will be shown that Eqs. (10a)-(10c) are quantitatively very accurate (For e.g., see Fig. 5).

From Fig. 3, it can be seen that the behavior of $|G|$ is step-like with its magnitude abruptly increasing with $\gamma$ before saturating. However, since $\gamma$ is proportional to $z / z_{0}$ (from Eq. (10c)), it implies that $z_{0}$ may be viewed as a critical-length parameter beyond which $\left|A_{T H z}(\Omega, z)\right|$ becomes significant. From Eq. (3b) and Eq. (10c), $z_{0} \approx c \Delta n^{-1} \sqrt{\tau \tau_{T L}}$. Therefore, optimal interaction lengths are expected to be shorter for smaller $\tau, \tau_{T L}$. These deductions are in agreement with the numerical simulations in the next section (See Fig. 4(d)).

Next, in the inset (i) of Fig. 3, we plot the phase $\angle G$. It can be seen that both magnitude and phase of $G$ show oscillations for small values of $\gamma$, before stabilizing at larger values. Since $\gamma$ is also a function of frequency, ripples in $|G|$ and $\angle G$ translate to ripples in the spectral magnitude and phase of $A_{T H z}(\Omega, z)$.

Finally, for the same $z, \gamma$ values decrease with increasing $\Omega$. Therefore, in conjunction with the prefactor $\Omega$ in Eq. (10a), the spectral magnitude $A_{T H z}(\Omega, z)$ can be expected to show a ramp-like shape, i.e. increasing with frequency for $\Omega<\Omega_{0}$ and then rapidly falling off for $\Omega>\Omega_{0}$. This can be confirmed by inspecting the terahertz spectra in Fig. 5 .

\subsection{Efficiency trends}

The conversion efficiency $\eta$ is obtained in general using Eq. (11a). It was previously shown that the upper limit of $\left|A_{T H z}(\Omega, z)\right|$ from Eq. (10a) is given by Eq. (8). Employing this fact, an 


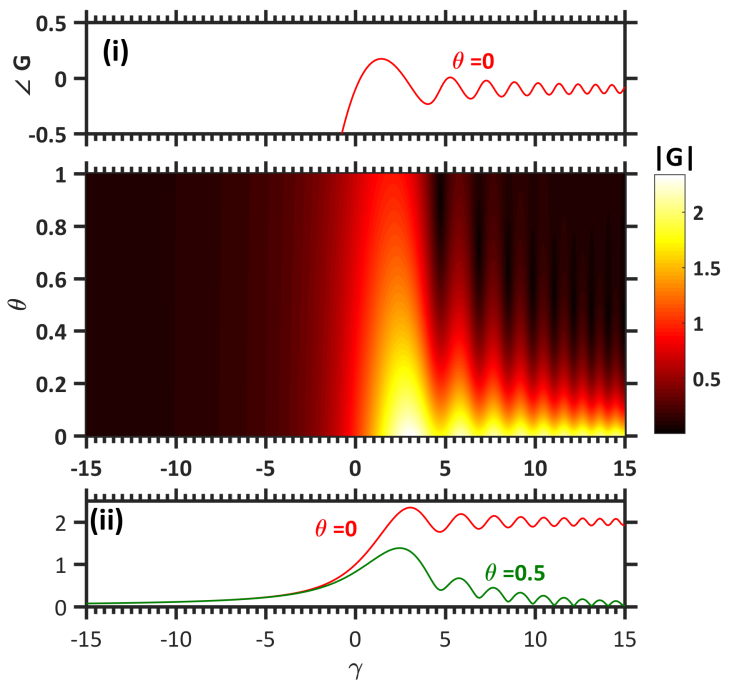

Fig. 3. Magnitude and phase of $G$ (Eq. (10b)) with $-L / 2 \rightarrow-\infty$ as a function of normalized variables $\gamma, \theta$ and $z_{0}$ (Eq. (10c)) which simultaneously account for the effects of $\tau_{T L}, \tau$, propagation distance $z$ and absorption coefficient $\alpha(\Omega)$. The phase and magnitude oscillations in $G$ manifest as spectral magnitude and phase oscillations in $A_{T H z}(\Omega, z)$.

upper-bound for $\eta$ based on an undepleted approximation is presented in Eq. (11b).

$$
\begin{gathered}
\eta(z)=\pi c \varepsilon_{0} \int_{0}^{\infty} n_{T H z}\left|A_{T H z}(\Omega, z)\right|^{2} d \Omega / F_{\text {pump }} \\
\eta \leq \frac{\pi \chi_{b}^{2} F_{\text {pump }} \Omega_{0}^{2} \tau_{T L}}{2 \sqrt{2} c \varepsilon_{0} n_{T H z} \Delta n^{2} n_{g}^{2}}
\end{gathered}
$$

From Eq. (11b), the proportionality of $\eta$ to $\tau_{T L}$ delineates that the conversion efficiency is inversely proportional to the generated terahertz bandwidth. This is understandable given that a larger bandwidth, when apportioned over the same length, translates to less interaction length per generated terahertz frequency. Thus, it is presumable that the conversion efficiency that is generated from such structures would be lesser than that of multi-cycle generation with periodically-poled structures. On the other hand, it can be seen that despite this limitation, the conversion efficiency for moderate terahertz pulse durations breaches the percent level. Furthermore, the conversion efficiency is inversely proportional to the difference in terahertz phase and optical group refractive index $\Delta n$. In light of this, KTP with smaller values of $\Delta n$ and potentially larger damage fluence thresholds may offer similar if not superior performance in relation to lithium niobate.

\section{Simulation results}

In this section, we present simulation results incorporating the full effects of dispersion, absorption, exact spatial variation of $\chi^{(2)}(z)$ (Eq. (5)) as well as pump depletion (or cascading effects). We first obtain optimal parameters via a numerical solution in Eq. (6) in the undepleted limit 

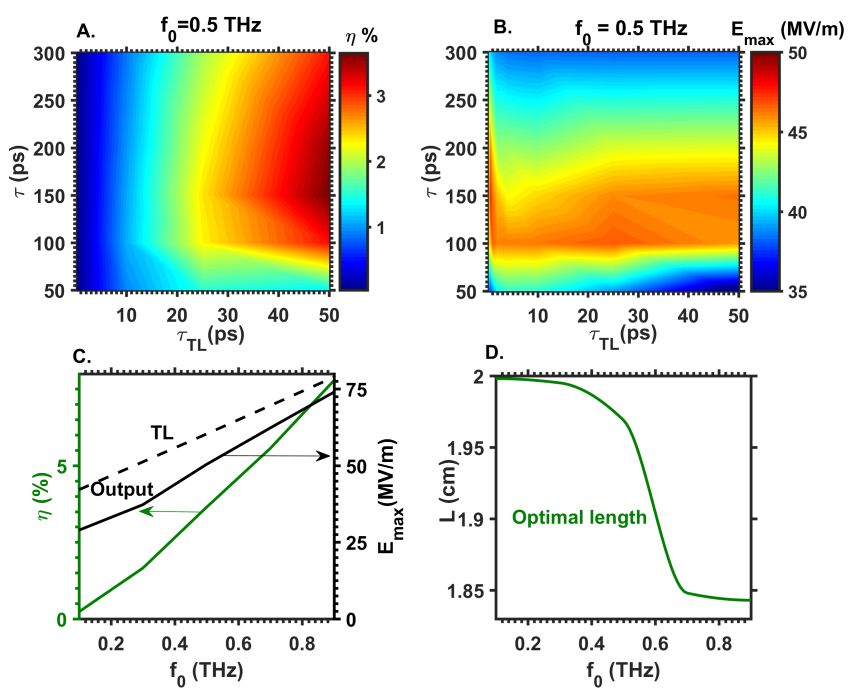

Fig. 4. (a) Conversion efficiency $\eta$ as a function of chirped-pulse duration $\tau$ and transformlimited duration $\tau_{T L}$ for $0.5 \mathrm{THz}$ in lithium niobate at $\mathrm{T}=80 \mathrm{~K}$. Increasing $\eta$ for narrower terahertz bandwidths is predicted, reaching about $4 \%$ for $\tau_{T L}=50 \mathrm{ps}$. (b) Corresponding electric field strengths in free space as a function of $\tau, \tau_{T L}$, showing collimated field strengths on the order of $50 \mathrm{MV} / \mathrm{m}$. Focused field strengths are then expected to be $\gg 100 \mathrm{MV} / \mathrm{m}$ for $\sim \mathrm{cm}^{2}$ crystal apertures. (c) Optimal $\eta$ and peak electric field strengths as a function of terahertz frequency, depicting an increasing trend and proximity of the peak fields to the corresponding transform-limited values. The optimal values occur for $\tau_{T L}=50 \mathrm{ps}$ and $\tau \approx 2 \Delta n c^{-1} \alpha^{-1} \approx 150 \mathrm{ps}$. (d) Optimal interaction lengths decrease with frequency, in agreement with increasing absorption coefficients with terahertz frequency.

and then verify functionality of the approach in the presence of cascading effects (or depleted calculations). Conversion efficiencies $\eta$ are evaluated using Eq. (11a). While the simulation results are carried out for cryogenically-cooled lithium niobate $(T=80 \mathrm{~K})$, the trends can be considered to apply to other material systems. Once the pump field is defined by Eqs. (3a)-(3c), the poling profile is uniquely defined by Eq. (5). The bulk second-order nonlinear susceptibility is assumed to be $\chi_{b}^{(2)}=336 \mathrm{pm} / \mathrm{V}$. The material disperion in the NIR region is obtained from [56]. The material dispersion for lithium niobate in the terahertz region is obtained from [57] while terahertz absorption is obtained from [58]. The total pump fluence that may be impinged on the crystals, is assumed to be limited by laser-induced damage according to the following expression for fluence, $F_{\text {pump }}=0.85(\tau / 100 \mathrm{ps})^{0.5} \mathrm{Jcm}^{-2}$ [43], where $\tau$ is the $e^{-2}$ pulse duration.

\subsection{Efficiency and peak fields}

We study the dependence of the conversion efficiency $(\eta)$ and peak electric field $\left(E_{\max }\right)$ on the pump parameters $\tau, \tau_{T L}$ for $f_{0}=0.5 \mathrm{THz}$ in Figs. 4(a) and 4(b) respectively.

Firstly, in Fig. 4(a), in line with Eq. (11b), we see an increase in conversion efficiency $\eta$ with increasing $\tau_{T L}$. As previously discussed, this may be understood by noting that when the same length is apportioned over a larger bandwidth, the coherent build up of each terahertz spectral component is limited. Therefore for small $\tau_{T L}, \eta$ is limited to a fraction of a percent but for larger $\tau_{T L}$, the conversion efficiencies breach the percent mark comfortably. However, contrary to Eq. (11b), the increase in conversion efficiency with $\tau_{T L}$ is not linear as Eq. (11b) represents the ideal limit.

There also exists an optimal value of $\tau$ for a given $\tau_{T L}$ in Fig. 4(a). This may be understood as 
follows. Firstly, the bandwidth of the generated terahertz pulse is limited by $\tau_{T L}^{-1}$, or the range of beat frequencies between the two optical pump pulses. The length of the aperiodically poled crystal is optimized when the phase-matched bandwidth is similar to the bandwidth of the pump pulse. Based on Eq. (4), this translates to $b \Delta n c^{-1} L_{o p t} \approx \tau_{T L}^{-1}$. However, since $b=\left(\tau \tau_{T L}\right)^{-1}$ from Eq. (3b), one obtains a condition for the optimal crystal length as $L_{o p t} \approx c \tau \Delta n^{-1}$ for a given chirped-pulse duration $\tau$.

The maximum interaction length is in turn limited by the terahertz absorption coefficient. Thus, $L_{o p t} \approx 2 \alpha^{-1}$ and consequently $\tau_{o p t} \approx 2 \alpha^{-1} \Delta n c^{-1}$. As the pump bandwidth $\tau_{T L}$ increases, the absorption length reduces by virtue of spanning larger frequencies and hence both the optimal chirped-pulse duration and crystal length drop. The first trend is evident in Fig. 4(a).

In Fig. 4(b), the peak electric field strengths as a function of $\tau, \tau_{T L}$ are plotted. Since the emergent terahertz pulses are compressed, the peak-field strengths are proportional to $\eta / \tau_{T L}$. Therefore, for small $\tau_{T L}$, the peak electric field strengths are large despite low conversion efficiencies. After a minor drop for intermediate values of $\tau_{T L}$, the peak-field strengths tend to stabilize or saturate. The maximum field strengths for collimated beams in free space are on the order of $50 \mathrm{MV} / \mathrm{m}$. However, if one uses $\mathrm{cm}^{2}$-aperture crystals, focused field strengths of several hundred $\mathrm{MV} / \mathrm{m}$ are indeed feasible.

Figure 4(c) delineates the global optima of conversion efficiencies and peak-field strengths (w.r.t to parameters $\tau, \tau_{T L}$ ) for various central terahertz frequencies $f_{0}$. Figure 4 (d) depicts the corresponding optimal crystal lengths. Based on the trends in Fig. 4(a), the optimal value of $\tau_{T L}$ occurs in the many-cycle limit, i.e. for $\tau_{T L}=50 \mathrm{ps}$ or at the edge of the parameter space used for $\tau_{T L}$. The corresponding optimal value of $\tau \approx 2 \alpha^{-1} \Delta n c^{-1} \approx 150 \mathrm{ps}$.

In Fig. 4(c), the conversion efficiency (green) shows an increasing trend with frequency, albeit only linearly and not quadratically owing to the effects of increasing absorption with terahertz frequency. The maximum electric field strengths (black) also increase with frequency and are very close to the transform-limited values (black-dashed), further depicting the efficacy of the present mechanism in achieving compression. Figure 4(d) depicts the optimal interaction lengths as a function of frequency. Here, the trends of decreasing optimal lengths are consistent with increasing absorption coefficients with terahertz frequency.

\subsection{Spectra, phase and temporal formats}

In this section, we present terahertz spectra , phase and temporal electric field traces for various values of $\tau_{T L}$. In each case, the terahertz frequency was $0.5 \mathrm{THz}$ for optimal values of $\tau \approx 150 \mathrm{ps}$ obtained from the previous section.

In Fig. 5(a), we present the spectrum for $\tau_{T L}=0.5 \mathrm{ps}$. The spectral profile in Fig. 5(a) is clearly broadband spanning 0.2 to $0.8 \mathrm{THz}$. The increasing ramp-like shape and ripples are in line with the discussion following Fig. 3 as seen in the analytic calculations (red-dashed) from Eq. (10a). Furthermore, the analytic calculations from Eqs. (10a)-(10c) are in agreement with numerical simulations based on Eq. (6).

In Fig. 5(b), the group delay (GD) of the terahertz pulse is plotted as obtained from analysis (Eqs. (10a-10c)) as well as simulations (Eq. (6)). In addition, we plot the uncompensated group delay $\left(\Omega-\Omega_{0}\right) /\left(2 b+8 \tau^{-4} / b\right)$ (See derivative of the first phase term within brackets of Eq. (8)) to delineate the extent of phase compensation. As evident from Fig. 5(b), the linear group delay (black-dashed) has been roughly flattened (save for ripples, which are expected based on Eq. (10a)).

As a result, in Fig. 5(c), we find that the transform-limited terahertz transient (red) is very close to the output from the structure (green) and constitutes a single-cycle terahertz pulse with a duration proportional to $\tau_{T L}=0.5$ ps. Similarly, Figs. 5(d)-5(f) and Figs. 5(g)-5(i) represent the corresponding properties for $\tau_{T L}=4 \mathrm{ps}$ and $\tau_{T L}=25 \mathrm{ps}$ respectively, which both produce terahertz pulses (green) close to transform-limited values (red). 

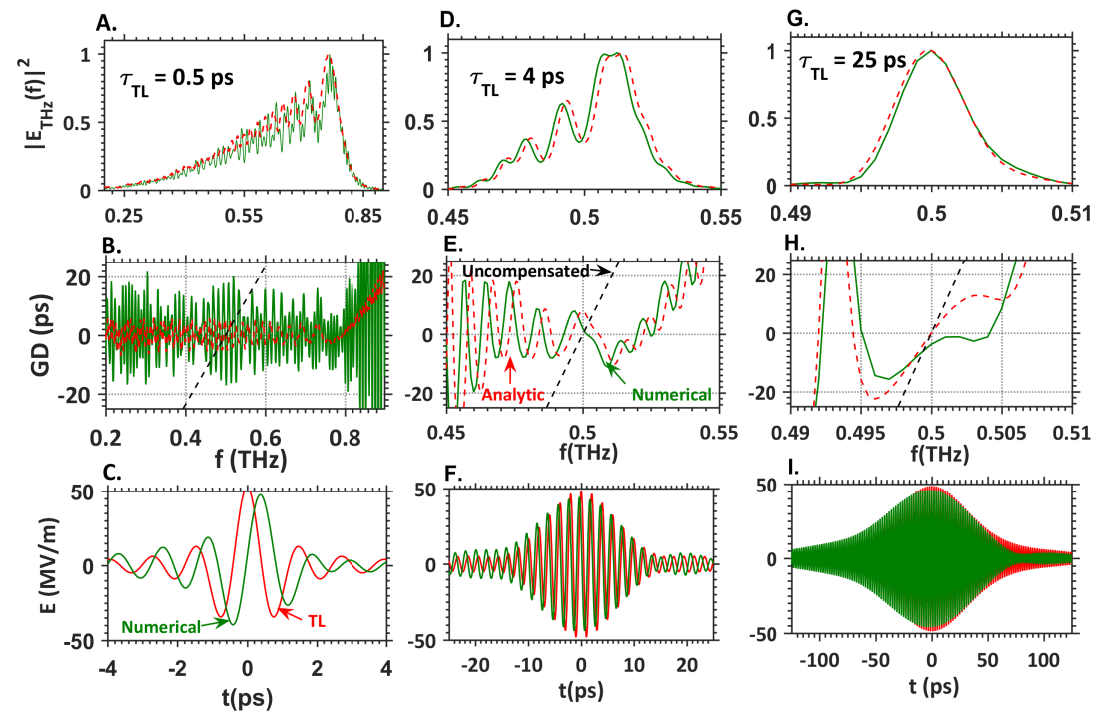

Fig. 5. Terahertz spectra and group delay (GD) obtained from simulations (Eq. (6), green) and analytic calculations (Eq. (10a), red-dashed) along with electric field output traces obtained from simulations only (green) and corresponding transform-limited values (red-solid). The uncompensated GD due to the relative chirp between pump pulses is also shown (black-dashed). For (a)-(c) $\tau_{T L}=0.5 \mathrm{ps}$,(d)-(f) $\tau_{T L}=4 \mathrm{ps}$ and for (g)-(i) $\tau_{T L}=25 \mathrm{ps}$. In all cases, the generated pulses are very close to the transform-limited output. The ripples in phase and spectra maybe rectified by apodization.

\subsection{Cascading effects}

In this section, we resort to simulations in the depleted limit, i.e. including cascading effects based on the numerical model presented in [43]. The calculations consider cascading effects, full material dispersion in the NIR frequency range [56], terahertz frequency range [57] as well as terahertz absorption [58] at $T=80 \mathrm{~K}$. The remaining parameters are the same as in previous sections and are taken from [43]. We find that despite drastic spectral broadening of the pump, the efficacy of compression is still maintained and efficiencies are in agreement with calculations in previous sections.

In Fig. 6(a), the optical spectrum corresponding to two pulses separated by a frequency of $0.5 \mathrm{THz}$, with parameters $\tau_{T L}=25 \mathrm{ps}$, and $\tau=150 \mathrm{ps}$ is depicted. The choice of these parameters is based on the fact that the expected conversion efficiencies fall in a regime where cascading effects are expected to be significant due to large values of $\eta$ (See Fig. 4(a)). For shorter $\tau_{T L}$, the similarity to undepleted calculations would increase owing to lower expectations of conversion efficiency (See Fig. 4(a)). The extreme values of the poling period for this structure range between $241.8 \mu \mathrm{m}$ at the beginning of the crystal to $238.3 \mu \mathrm{m}$ at the end. This is within the tolerance offered by current technology [48], which is $<0.1 \mu \mathrm{m}$.

Since the energy in the narrowband pulse and broadband chirped pulse are equal, their relative strengths in the spectral domain are dissimilar as is evident from the inset on the right in Fig. 6(a). Note, how this inset shows differing linewidths. The inset on the left plots conversion efficiency versus length and shows that the conversion efficiency is similar, albeit higher compared to that obtained via undepleted calculations. The higher conversion efficiency of $4 \%$ is due to the increase in pump intensity resulting from dramatic spectral broadening.

In Figs. 6(b)-6(d), we observe a significant red-shift of the optical spectrum due to cascading effects, which is responsible for the high conversion efficiency in the first place. However, due to 

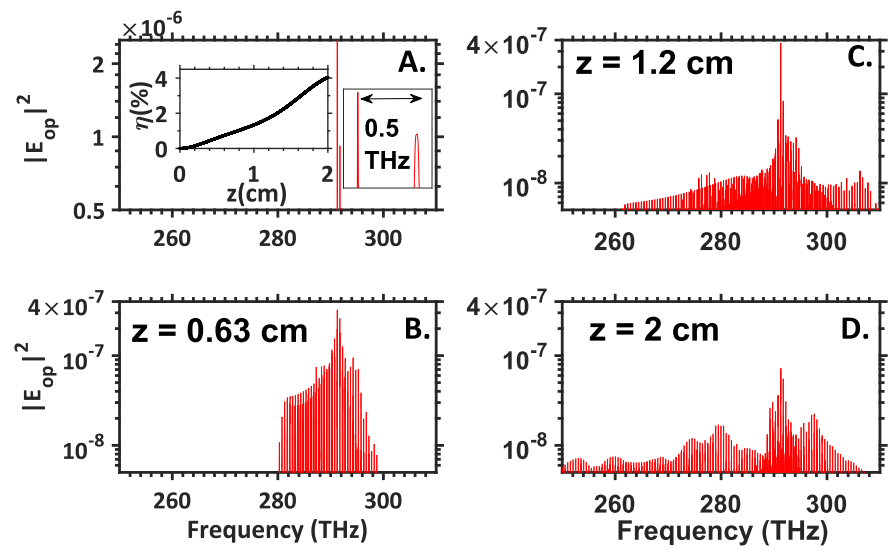

Fig. 6. Evolution of the optical pump spectrum as a function of distance. (a) Two 'lines' separated by $0.5 \mathrm{THz}$ (see inset on right), one of which is broader due to a linewidth defined by $\tau_{T L}=25 \mathrm{ps}$. The inset on the left shows conversion efficiency approaching $4 \%$ at $T=80 \mathrm{~K}$. The efficiency is larger than the corresponding undepleted simulaiton due to an increase in optical pulse intensity which arises from spectral broadening. (b)-(d) A continuous red-shift is obtained due to the beating of the lines. This red-shift is responsible for the high conversion efficiencies that are observed. Blue-shift is also obtained since sum and difference frequency processes are both phase matched due to the small terahertz frequency.

the collinear geometry of this system, a spatio-temporal break-up of the pulse does not occur. This is contrary to the case of tilted pulse fronts.

Since, the strength of the two input lines differ, the situation is similar to that of a cascaded terahertz parametric amplifier [59]. Comparing Figs. 6(a)-6(d), the spectrum shows an increasing red-shift with distance, accompanied also with some blue-shift due to symmetry in the phase matching of sum and difference frequency generation that results from the small value of the terahertz frequency. This is similar to that observed in $[59,60]$.

In Fig. 7(a), we plot the simulated terahertz field output and compare it to the transformlimited terahertz field (obtained by assuming a flat phase across the simulated spectrum). The peak-field strength of the transform-limited field (red) is about $75 \mathrm{MV} / \mathrm{m}$ (slightly larger than the transform-limited value of $60 \mathrm{MV} / \mathrm{m}$ in Fig. 5(i) due to the increase in terahertz efficiency by cascading effects), while the output from the structure (green) is close but smaller at $55 \mathrm{MV} / \mathrm{m}$ (similar to peak-field strengths obtained from undepleted calculations in Fig. 5(i). The duration of the two pulses are quite similar, indicating effective compression.

Figure 7(b) depicts the group delay obtained numerically while considering cascading effects (green) and analytically under undepleted approximations (Eq. (10a), red-dashed) in comparison to the uncompensated group delay of the pump pulse input (black-dashed). Clearly, the chirp on the pump input has been significantly reduced by the structure (shown by brown arrows) as evident in both simulations and analytic calculations. However, for frequencies $>0.502 \mathrm{THz}$, the undepleted calculations suggest better compression.

However, since most of the energy in the spectrum is contained within the region where undepleted and depleted calculations agree (red, inset of Fig. 7(b)), effective compression is nevertheless obtained. To delineate how broadband the generated terahertz spectrum is, we also plot the spectrum of a narrowband terahertz pulse corresponding to $\tau=150 \mathrm{ps}$ (blue) in the inset.

The difference in group delay between depleted and undepleted calculations is due to the 

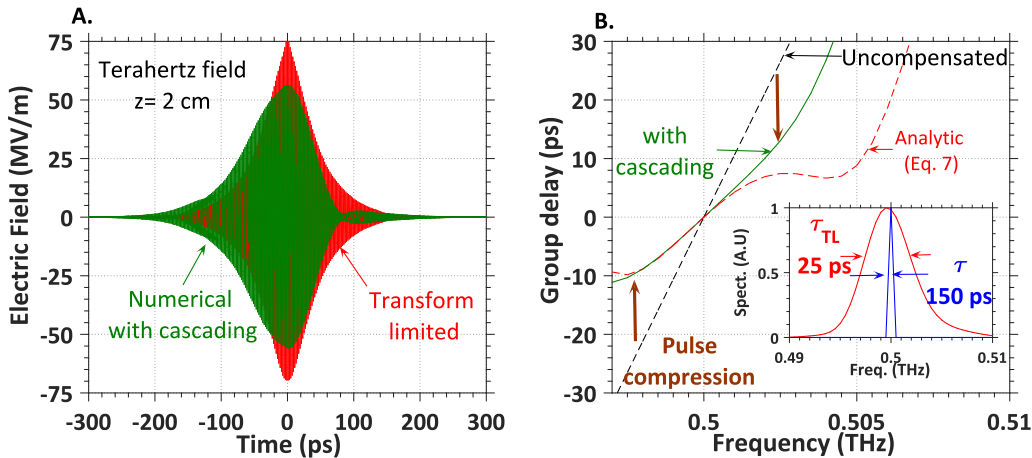

Fig. 7. (a) Traces of the terahertz electric field obtained from depleted simulations accounting for cascading (green) and the corresponding transform-limited terahertz pulse(red). The output from the structure is similar to the transform-limited value and has a peak-field strength similar to that predicted from undepleted calculations. (b) Group delay of compressed pulses obtained from depleted simulations (green) and undepleted analysis (red-dashed) show effects of phase compensation in relation to the chirp present in the pump input (black-dashed). The analytic calculations show superior compression for $>.502 \mathrm{THz}$, due to the effects of dispersion arising from cascading. Most of the generated spectrum (inset,red-solid) lies in a range where the undepleted and depleted calculations are in agreement, which results in effective compression. The spectrum of a narrowband pulse corresponding to $\tau=150 \mathrm{ps}$ (inset,blue-solid) is shown to delineate the large bandwidth of the generated terahertz pulse. For lower $\eta$, cascading effects would be less important and the efficacy of compression would approach that predicted by undepleted calculations.

change in optical group refractive index encountered by the broadened pump spectrum. For such broadband spectra, higher-order dispersion is also significant. This renders the generated poling profile less effective. Since, the extent of broadening is efficiency dependent, the disparity between depleted and undepleted calculations can be expected to reduce for more broadband terahertz generation (e.g Fig. 5(a)), where the efficiencies are anticipated to be lower.

In the high efficiency regime delineated in Figs. 6 and 7, the compression may be further improved by numerical optimization of the poling profile. This could also be useful in mitigating effects of higher-order dispersion and would be the scope of future work.

In any case, it is worthwhile to note that the poling profile conceived analytically in the absence of depletion is highly effective, even in the presence of cascading effects, producing conversion efficiencies $\approx 4 \%$. This also suggests that the device maybe robust to spurious higher-order dispersion.

\section{Conclusion}

In conclusion, a new method to generate broadband terahertz pulses using a combination of a non-uniform sequence of pulses and aperiodically poled crystals was shown to produce compressed pulses with large peak-field strengths and high efficiencies. The non-uniform sequence of pulses could be generated by the superposition of a chirped and transform-limited pulse separated by the desired terahertz frequency. The approach is particularly efficacious for generating few to tens-of-cycles terahertz pulses, yielding conversion efficiencies of $\approx 4 \%$. Analysis in the undepleted limit along with simulations in undepleted and depleted limits were presented. The simulations validated the efficacy of the approach. In the presence of dramatic cascading, the efficacy of compression only reduces marginally but this may be further improved by numerical optimization of the poling profile. The presented work could potentially be of high value for terahertz-driven electron acceleration and coherent X-ray generation. 


\section{Funding}

Air Force Office of Scientific Research (AFOSR) (A9550-12-1-0499); European Research Council (609920); Center for Free-Electron Laser Science at DESY.

\section{Acknowledgments}

The authors would like to thank Prof. Erich P. Ippen and Dr. Phillip D. Keathley for useful comments.

\section{References}

1. P. K. Mishra, O. Vendrell, and R. Santra, "Ultrafast energy transfer to liquid water by sub-picosecond high-intensity terahertz pulses: An ab initio molecular dynamics study," Angewandte Chemie Int. Ed. 52, 13685-13687 (2013).

2. P. K. Mishra, O. Vendrell, and R. Santra, "Ultrafast energy transfer from solvent to solute induced by subpicosecond highly intense thz pulses,” The J. Phys. Chem. B 119, 8080-8086 (2015).

3. T. Kampfrath, K. Tanaka, and K. A. Nelson, "Resonant and nonresonant control over matter and light by intense terahertz transients," Nat. Photonics 7, 680-690 (2013).

4. M. Eisele, T. L. Cocker, M. A. Huber, M. Plank1, L. Viti, D. Ercolani, L. Sorba, M. S. Vitiello, and R. Huber, "Ultrafast multi-terahertz nano-spectroscopy with sub-cycle temporal resolution," Nat. Photonics 8, 841-845 (2014).

5. T. L. Cocker, M. A. Huber, M. Eisele, M. Plankl, L. Viti, R. E. Marvel, F. Sandner, F. Mooshammer, T. Korn, C. Schüller, D. Ercolani, L. Sorba, R. F. Haglund, M. S. Vitiello, and R. Huber, "Taking sub-cycle thz nanoscopy to the limits," in Conference on Lasers and Electro-Optics, (Optical Society of America, 2016), p. SW1L.4.

6. C. Ropers, "Electrons catch a terahertz wave," Science 352, 410-411 (2016).

7. C. Kealhofer, W. Schneider, D. Ehberger, A. Ryabov, F. Krausz, and P. Baum, "All-optical control and metrology of electron pulses," Science 352, 429-433 (2016).

8. L. Pálfalvi, J. A. Fülöp, G. Tóth, and J. Hebling, "Evanescent-wave proton postaccelerator driven by intense thz pulse,” Phys. Rev. ST Accel. Beams 17, 031301 (2014).

9. E. A. Nanni, W. R. Huang, K.-H. Hong, K. Ravi, A. Fallahi, G. Moriena, R. J. Dwayne Miller, and F. X. Kärtner, "Terahertz-driven linear electron acceleration," Nat. Commun. 6, 8486 (2015).

10. W. R. Huang, A. Fallahi, X. Wu, H. Cankaya, A.-L. Calendron, K. Ravi, D. Zhang, E. A. Nanni, K.-H. Hong, and F. X. Kärtner, “Terahertz-driven, all-optical electron gun,” Optica 3, 1209-1212 (2016).

11. A. Fallahi, M. Fakhari, A. Yahaghi, M. Arrieta, and F. X. Kärtner, "Short electron bunch generation using single-cycle ultrafast electron guns," Phys. Rev. Accel. Beams 19, 081302 (2016).

12. D. Zhang, A. Fallahi, M. Hemmer, X. Wu, M. Fakhari, Y. Hua, H. Cankaya, A.-L. Calendron, L. E. Zapata, N. H. Matlis, and F. X. Kärtner, "Segmented terahertz electron accelerator and manipulator (steam)," Nat. Photonics 12, 336-342 (2018).

13. F. Lemery, K. Floettmann, P. Piot, F. Kärtner, and R. Aßmann, "Synchronous acceleration with tapered dielectric-lined waveguides," Phys. Rev. Accel. Beams 21, 051302 (2018).

14. F. Kärtner, F. Ahr, A.-L. Calendron, H. Çankaya, S. Carbajo, G. Chang, G. Cirmi, K. Dörner, U. Dorda, A. Fallahi, A. Hartin, M. Hemmer, R. Hobbs, Y. Hua, W. Huang, R. Letrun, N. Matlis, V. Mazalova, O. Mücke, E. Nanni, W. Putnam, J. Ravi, F. Reichert, I. Sarrou, X. Wu, A. Yahaghi, Y. Hong, L. Zapata, D. Zhang, C. Zhou, R. Miller, K. Berggren, H. Graafsma, A. Meents, R. Assmann, H. Chapman, and P. Fromme, "Axsis: Exploring the frontiers in attosecond X-ray science, imaging and spectroscopy," Nucl. Instruments Methods Phys. Res. Sect. A: Accel. Spectrometers, Detect. Assoc. Equip. 829, 24-29 (2016).

15. N. T. Yardimci, S. H. Yang, C. W. Berry, and M. Jarrahi, "High-power terahertz generation using large-area plasmonic photoconductive emitters," IEEE Transactions on Terahertz Sci. Technol. 5, 223-229 (2015).

16. S. H. Gold and G. S. Nusinovich, "Review of high-power microwave source research," Rev. Sci. instruments $\mathbf{6 8}$, 3945-3974 (1997).

17. G. Gallerano and S. Biedron, "Overview of terahertz radiation sources," in Proceedings of the 2004 FEL Conference, (2004), 1, pp. 216-221.

18. M. Cronin-Golomb, "Cascaded nonlinear difference-frequency generation of enhanced terahertz wave production," Opt. Lett. 29, 2046-2048 (2004).

19. W. R. Huang, S.-W. Huang, E. Granados, K. Ravi, K.-H. Hong, L. E. Zapata, and F. X. Kärtner, "Highly efficient terahertz pulse generation by optical rectification in stoichiometric and cryo-cooled congruent lithium niobate," $\mathrm{J}$ Mod. Opt. 62, 1486-1493 (2015).

20. C. Vicario, A. V. Ovchinnikov, S. I. Ashitkov, M. B. Agranat, V. E. Fortov, and C. P. Hauri, "Generation of 0.9-mj thz pulses in dstms pumped by a cr:mg2sio4 laser,” Opt. Lett. 39, 6632-6635 (2014)

21. J. Lu, H. Y. Hwang, X. Li, S.-H. Lee, O.-P. Kwon, and K. A. Nelson, "Tunable multi-cycle thz generation in organic crystal hmq-tms," Opt. Express 23, 22723-22729 (2015). 
22. J. Hebling, G. Almasi, I. Z. Kozma, and J. Kuhl, "Velocity matching by pulse front tilting for large-area thz-pulse generation," Opt. Express 10, 1161-1166 (2002).

23. H. Hirori, A. Doi, F. Blanchard, and K. Tanaka, "Single-cycle terahertz pulses with amplitudes exceeding $1 \mathrm{mv} / \mathrm{cm}$ generated by optical rectification in linbo 3," Appl. Phys. Lett. 98, 091106 (2011).

24. J. Fülöp, L. Pálfalvi, S. Klingebiel, G. Almási, F. Krausz, S. Karsch, and J. Hebling, "Generation of sub-mj terahertz pulses by optical rectification," Opt. Lett. 37, 557-559 (2012).

25. Z. Chen, X. Zhou, C. A. Werley, and K. A. Nelson, "Generation of high power tunable multicycle teraherz pulses," Appl. Phys. Lett. 99, 071102 (2011).

26. J. A. Fülöp, Z. Ollmann, C. Lombosi, C. Skrobol, S. Klingebiel, L. Pálfalvi, F. Krausz, S. Karsch, and J. Hebling, "Efficient generation of thz pulses with 0.4 mj energy," Opt. Express 22, 20155-20163 (2014).

27. K. Ravi, W. R. Huang, S. Carbajo, X. Wu, and F. X. Kärtner, "Limitations to thz generation by optical rectification using tilted pulse fronts," Opt. Express 22, 20239-20251 (2014).

28. F. Blanchard, X. Ropagnol, H. Hafez, H. Razavipour, M. Bolduc, R. Morandotti, T. Ozaki, and D. Cooke, "Effect of extreme pump pulse reshaping on intense terahertz emission in lithium niobate at multimillijoule pump energies," Opt. Lett. 39, 4333-4336 (2014).

29. K. Ravi, W. R. Huang, S. Carbajo, E. A. Nanni, D. N. Schimpf, E. P. Ippen, and F. X. Kärtner, "Theory of terahertz generation by optical rectification using tilted-pulse-fronts,” Opt. Express 23, 5253-5276 (2015).

30. C. Lombosi, G. Polónyi, M. Mechler, Z. Ollmann, J. Hebling, and J. Fülöp, "Nonlinear distortion of intense thz beams," New J. Phys. 17, 083041 (2015).

31. B. K. Ofori-Okai, P. Sivarajah, W. R. Huang, and K. A. Nelson, "Thz generation using a reflective stair-step echelon," Opt. Express 24, 5057-5068 (2016)

32. K. Ravi, B. K. Ofori-Okai, P. Sivarajah, W. R. Huang, F. X. Kärtner, and K. A. Nelson, "Circumventing limitations of tilted-pulse-front terahertz generation using a stair-step echelon," in Progress in Electromagnetic Research Symposium (PIERS), (IEEE, 2016), pp. 3917-3918.

33. K. Ravi, B. Ofor-Okai, P. Sivarajah, W. R. Huang, K. A. Nelson, and F. X. Kärtner, "Terahertz generation by beamlet superposition," arXiv preprint arXiv:1810.09118 (2018).

34. Y. Avetisyan, A. Makaryan, V. Tadevosyan, and M. Tonouchi, "Design of a multistep phase mask for high-energy terahertz pulse generation by optical rectification," J. Infrared, Millimeter, Terahertz Waves 38, 1439-1447 (2017).

35. L. Pálfalvi, G. Tóth, L. Tokodi, Z. Márton, J. A. Fülöp, G. Almási, and J. Hebling, "Numerical investigation of a scalable setup for efficient terahertz generation using a segmented tilted-pulse-front excitation," Opt. Express $\mathbf{2 5}$, 29560-29573 (2017).

36. G. Polonyi, M. I. Mechler, J. Hebling, and J. A. Fülöp, “Prospects of semiconductor terahertz pulse sources," IEEE J. Sel. Top. Quantum Electron. 23, 1-8 (2017).

37. P. S. Nugraha, G. Krizsán, C. Lombosi, L. Pálfalvi, G. Tóth, G. Almási, J. A. Fülöp, and J. Hebling, "Demonstration of a tilted-pulse-front pumped plane-parallel slab terahertz source," arXiv preprint arXiv:1808.06122 (2018).

38. Y. S. Lee, T. Meade, V. Perlin, H. Winful, T. Norris, and A. Galvanauskas, "Generation of narrow-band terahertz radiation via optical rectification of femtosecond pulses in periodically poled lithium niobate," Appl. Phys. Lett. 76 2505-2507 (2000).

39. K. L. Vodopyanov, "Optical generation of narrow-band terahertz packets in periodically-inverted electro-optic crystals: conversion efficiency and optimal laser pulse format," Opt. Express 14, 2263-2276 (2006).

40. I. Tomita, H. Suzuki, H. Ito, H. Takenouchi, K. Ajito, R. Rungsawang, and Y. Ueno, "Terahertz-wave generation from quasi-phase-matched gap for $1.55 \mu$ m pumping," Appl. Phys. Lett. 88, 071118 (2006).

41. T. Hattori and K. Takeuchi, "Simulation study on cascaded terahertz pulse generation in electro-optic crystals," Opt. Express 15, 8076-8093 (2007).

42. K. L. Vodopyanov, "Optical thz-wave generation with periodically-inverted gaas," Laser \& Photonics Rev. 2, 11-25 (2008).

43. K. Ravi, D. N. Schimpf, and F. X. Kärtner, "Pulse sequences for efficient multi-cycle terahertz generation in periodically poled lithium niobate," Opt. Express 24, 25582-25607 (2016).

44. A. Yahaghi, K. Ravi, A. Fallahi, and F. Kärtner, "Designing chirped aperiodically poled structures for high-energy single-cycle terahertz generation,” J. Opt. Soc. Am. B 34, 590-600 (2017).

45. K. Ravi and F. X. Kärtner, "Generating compressed broadband terahertz pulses using aperiodically poled electro-optic crystals," arXiv preprint arXiv:1710.07843 (2017).

46. K. Ravi and F. X. Kärtner, "Simultaneous generation and compression of broadband terahertz pulses in aperiodically poled crystals," in CLEO: Science and Innovations, (Optical Society of America, 2018), pp. SM4A-2.

47. Y. Avetisyan and M. Tonouchi, "Nearly single-cycle terahertz pulse generation in aperiodically poled lithium niobate," Preprints 2018120288 (2018)

48. H. Ishizuki and T. Taira, "Improvement of laser-beam distortion in large-aperture ppmgln device by using $\mathrm{x}$-axis czochralski-grown crystal," Opt. Express 22, 19668-19673 (2014).

49. L. E. Zapata, F. Reichert, M. Hemmer, and F. X. Kärtner, " $250 \mathrm{w}$ average power, $100 \mathrm{khz}$ repetition rate cryogenic yb:yag amplifier for opcpa pumping," Opt. Lett. 41, 492-495 (2016).

50. M. A. Arbore, O. Marco, and M. M. Fejer, "Pulse compression during second-harmonic generation in aperiodic quasi-phase-matching gratings," Opt. Lett. 22, 865-867 (1997).

51. M. Arbore, A. Galvanauskas, D. Harter, M. Chou, and M. Fejer, "Engineerable compression of ultrashort pulses by 
Optics EXPRESS

use of second-harmonic generation in chirped-period-poled lithium niobate," Opt. Lett. 22, 1341-1343 (1997).

52. A. Galvanauskas, D. Harter, M. A. Arbore, M. H. Chou, and M. M. Fejer, "Chirped-pulse-amplification circuits for fiber amplifiers, based on chirped-period quasi-phase-matching gratings," Opt. Lett. 23, 1695-1697 (1998).

53. P. Loza-Alvarez, D. T. Reid, P. Faller, M. Ebrahimzadeh, and W. Sibbett, "Simultaneous second-harmonic generation and femtosecond-pulse compression in aperiodically poled ktiopo4 with a rbtioaso4-based optical parametric oscillator," J. Opt. Soc. Am. B 16, 1553-1560 (1999).

54. L. J. Wong, A. Fallahi, and F. X. Kärtner, "Compact electron acceleration and bunch compression in thz waveguides," Opt. Express 21, 9792-9806 (2013).

55. J. L'huillier, G. Torosyan, M. Theuer, Y. Avetisyan, and R. Beigang, "Generation of thz radiation using bulk, periodically and aperiodically poled lithium niobate-part 1: Theory," Appl. Phys. B 86, 185-196 (2007).

56. D. H. Jundt, "Temperature-dependent sellmeier equation for the index of refraction, $\mathrm{n}$ e, in congruent lithium niobate," Opt. Lett. 22, 1553-1555 (1997).

57. L. Pálfalvi, J. Hebling, J. Kuhl, A. Peter, and K. Polgár, "Temperature dependence of the absorption and refraction of mg-doped congruent and stoichiometric linbo 3 in the thz range," J. Appl. Phys. 97, 123505 (2005).

58. J. A. Fülöp, L. Pálfalvi, M. C. Hoffmann, and J. Hebling, "Towards generation of mj-level ultrashort thz pulses by optical rectification,” Opt. Express 19, 15090-15097 (2011).

59. K. Ravi, M. Hemmer, G. Cirmi, F. Reichert, D. N. Schimpf, O. D. Mücke, and F. X. Kärtner, "Cascaded parametric amplification for highly efficient terahertz generation,” Opt. Lett. 41, 3806-3809 (2016).

60. M. Hemmer, G. Cirmi, K. Ravi, F. Reichert, F. Ahr, L. Zapata, O. D. Mücke, A.-L. Calendron, H. Çankaya, D. Schimpf, N. H. Matlis, and F. X. Kärtner, "Cascaded interactions mediated by terahertz radiation," Opt. Express 26, 12536-12546 (2018). 